\title{
Complutum
}

ISSN: 1131-6993

\section{Etnoarqueología del combustible: Una aproximación arqueológica a las interacciones sociales entre bosques, árboles y personas ${ }^{1}$}

\author{
Llorenç Picornell-Gelabert ${ }^{2}$
}

Recibido: 9 de septiembre de 2016 / Aceptado: 15 de febrero de 2017

Resumen. En este artículo expongo algunas reflexiones en torno al uso de la etnoarqueología como escenario para el estudio de las relaciones entre los humanos y su entorno. Partiendo de una revisión bibliográfica (que no pretende ser exhaustiva) y de diversos casos de estudio de mi trabajo de campo en Ghana y Guinea Equatorial, discuto como las relaciones entre personas y árboles para el uso de la madera - leña - como combustible constituye una arena social de interacción entre humanos y no-humanos. Analizo como estas prácticas cotidianas de aprovisionamiento y consumo energético en el bosque, lejos de ser prácticas monótonas y alejadas de toda creatividad social, se integran y participan de la materialización de las diversas formas en que las sociedades humanas perciben el entorno y lo socializan mediante la acción material sobre éste. De esta forma, se evidencian algunas discusiones que permiten (i) generar aproximaciones arqueológicas a este tipo de cuestiones relacionadas con las interacciones entre naturaleza y cultura; e (ii) integrar perspectivas, aproximaciones, prácticas y narrativas entre las llamadas arqueología cientifica (en especial referencia a la antracología, disciplina encargada del estudio de restos materiales de árboles y arbustos, carbón y madera, en yacimientos arqueológicos) y arqueología teórica o antropológica.

Palabras clave: Árboles; combustible; arqueobotánica; antracología; naturaleza-cultura.

\section{[en] Fuel Ethnoarchaeology. An Archaeological Approach to Social Interactions between Forests, Trees and People}

\begin{abstract}
In this article I depict some considerations about the use of ethnoarchaeology as a scene for the study of the relations between humans and the environment. On the basis of a bibliographical revision (which do not intend to be exhaustive) and from diverse cases of study from my fieldwork in Ghana and Equatorial Guinea, I discuss how relations between people and trees for the use of wood - firewood - as fuel constitutes an arena of social interaction between humans and non-humans. I analyze how these everyday practices of energetic supply and consumption in the forest, far from being monotonous practices away from social creativity, are integrated and participate in the materialization of the diverse forms in which human societies perceive the environment and socialize it through material action. In this sense, diverse discussions arise and allow (i) generating archaeological approaches to this kind of culture-nature interactions; and (ii) integrating perspectives, approaches, practices and narratives between the so-called "scientific archaeology" (with special emphasis on anthracology, the discipline studying the material remains of trees and shrubs - wood and charcoal - in archaeological sites) and "theoretical or anthropological archaeology".
\end{abstract}

Keywords: Trees; Fuel; Archaeobotany; Anthracology; Nature-Culture.

Sumario. 1. Árboles y ciencias sociales (introducción). 2. Prácticas invisibilizadas: aprovisionamiento y consumo de combustible. 3. Etnoarqueología de los árboles y el combustible. 3.1. Paleoeconomía del combustible. 3.2. Árboles, combustible y construcción del espacio social. 4. Visibilización de los árboles y de las prácticas cotidianas de consumo energético en las narrativas arqueológicas (reflexión final).

1 Este trabajo ha sido realizado gracias a una beca postdoctoral Beatriu de Pinós del AGAUR-Generalitat de Catalunya (2140BPA00216). El trabajo de campo del que proceden los ejemplos etnográficos ha sido realizado en Guinea Ecuatorial con financiación del MAEC-AECID, el Ministerio de Cultura (julio-octubre 2007-2008, agosto 2009; agosto 2010, enero-febrero 2012) y la Fondation Maison des Sciences de l'Homme (febrero-marzo 2013, beca Fernand Braudel); y en Ghana con financiación del Ministerio de Cultura (“Arqueología en la cuenca alta del Volta Blanco, Noreste de Ghana”, junio 2015 y febrero 2016).

2 UMR 7209 Archéozoologie, Archéobotanique: sociétés, pratiques et environnements.

Muséum National d'Histoire Naturelle - Centre National de la Recherche Scientifique - Sorbonne Universités.

ArqueoUIB Grup d'Investigació en Cultura Material i Gestió del Patrimoni Arqueològic. Universitat de les Illes Balears.

E-mail: tokelau24@gmail.com 
Cómo citar: Picornell-Gelabert, Ll. (2017): Etnoarqueología del combustible: Una aproximación arqueológica a las interacciones sociales entre bosques, árboles y personas. Complutum, 28(2): 325-339.

\section{1. Árboles y ciencias sociales (introducción)}

Desde hace décadas, tanto la antropología como la arqueología han explorado las relaciones entre humanos y los diversos elementos que componen lo que convenimos en llamar naturaleza. La profusión de trabajos y seminarios sobre relaciones entre animales humanos y no-humanos es especialmente fructífera en este sentido (Elden 2006; Ingolg 1988; Ito 2008; Philo y Wilbert 2000), así como lo son las diversas y numerosas aproximaciones al paisaje (p. ej. Wu 2010). Por lo que respeta al mundo vegetal, también se ha prestado atención a diversos aspectos sociales de las interacciones entre humanos y plantas, como el significado social de la recolección y producción de plantas comestibles (Bradley 2005: 121-144; Hastorf 2009; Palmer y Van der Veer 2002).

En cierto modo, todos estos campos de estudio parten del impulso que representa el cuestionamiento de la esencia y las relaciones entre dos grandes categorías de la modernidad: naturaleza y cultura. En antropología, la cuestión naturaleza/cultura ha dado pie a la construcción de un gran campo de estudio (Descola y Pálson 2001; Descola 2005; Ingold 2000; Latour 1991), aun siendo un terreno resbaladizo y polémico, pero extremadamente atractivo (Inglis y Bone 2008).

En este contexto, y en lo que refiere a la práctica arqueológica, el estudio de las relaciones socio-ambientales ha experimentado un cierto impulso. Diversas disciplinas arqueológicas, impulsadas por este creciente interés, han ido desarrollando múltiples métodos y técnicas de investigación centrados en generar nuevos conjuntos de datos ambientales a partir del estudio del registro arqueológico. La llamada arqueología ambiental ha experimentado desde la segunda mitad del siglo XX un importante desarrollo precisamente impulsada por la centralidad que las cuestiones ambientales han adquirido en las agendas arqueológicas. No obstante, este desarrollo se ha llevado a cabo a partir de una cierta incomunicación o desconexión entre la llamada arqueología científica, aquella que genera datos desde disciplinas de las ciencias naturales, y la arqueología teórica o antropológica, encargada de la interpreta- ción social e histórica del registro arqueológico (Luff y Rowley-Conwy 1994; Pollard y Bray 2007; Jones 2001).

En cualquier caso, tanto en la arqueología como en otras ciencias sociales, existe una determinada categoría del mundo natural cuya relevancia social no ha sido suficientemente explorada. En el apogeo de los estudios sobre las interacciones entre agentes humanos y nohumanos, los árboles nunca han adquirido una posición relevante en la interrogación de las implicaciones teóricas y metodológicas que éstos representan para las ciencias sociales. A pesar de la publicación de importantes obras en este sentido, es una constante en ellas la visibilización de la falta de atención a estos elementos naturales (Rival 1998; Jones y Cloke 2002; Watkins 2014). Esta invisibilidad de los árboles en la literatura de las ciencias sociales no parece una excepción en el caso de la arqueología, a pesar que desde hace décadas se desarrolla una disciplina de la llamada arqueología ambiental, la antracología, precisamente dedicada al estudio de los restos de estas plantas leñosas en el registro arqueológico.

Esta situación constituye el punto de partida de mi aproximación a la etnoarqueología como antracólogo, es decir, como arqueólogo especializado en el estudio de carbones y maderas procedentes de yacimientos arqueológicos. En este artículo voy a ilustrar como los estudios etnoarqueológicos proporcionan herramientas para construir una práctica arqueológica (y antracológica) que permita enriquecer las narrativas sobre las relaciones entre personas y árboles en el contexto de las complejas interacciones entre lo humano y lo no-humano (entre cultura y naturaleza). A pesar de que el objeto de estudio de los antracólogos, madera y carbón, representa esencialmente restos materiales de árboles del pasado en el registro arqueológico, la verdad es que la disciplina no ha sido ajena ni a la dicotomía naturaleza-cultura (científico-teórico) ni a la deficiente (si no inexistente) teorización del árbol como agente en las complejas redes de interacciones socioambientales.

En realidad, desde el inicio de la cristalización de la antracología como disciplina, la división heurística entre lo social y lo ambien- 
tal ha sido el combustible de la mayoría de los debates entre los especialistas. No obstante, desde hace unas décadas, la comunidad antracológica apela a la necesidad de construir nuevas aproximaciones al registro que partan de perspectivas menos sesgadas que permitan proporcionar conjuntos de datos y argumentos para la aproximación a las relaciones socio-ambientales (p. ej. Asouti y Austin 2005; Austin 2000; Piqué 1999; Picornell-Gelabert y Servera-Vives 2017). En este sentido, se ha propuesto la etnoarqueología como una de las vías para enriquecer los marcos de referencia de la disciplina, los objetos de estudio (diversos) y las cuestiones que dirigimos al registro antracológico. Esto debe ayudarnos en la tarea de unificar intereses (preguntas) y prácticas (aproximaciones al registro arqueológico) de la comunidad arqueológica en su conjunto (y no solo de la estrictamente antracológica). Esta unión entre intereses y prácticas arqueológicas constituye una estrategia para la construcción de nuevas narrativas arqueológicas que permitan visibilizar la relevancia social de los árboles (y no solo de los carbones como fósiles de árboles de ecosistemas pretéritos a reconstruir) y, a su vez, contribuir a enriquecer el análisis de las relaciones entre humanos y no-humanos en la literatura arqueológica.

Es en este contexto que sitúo el presente artículo. Sin pretender realizar una revisión exhaustiva de todos los trabajos etnoarqueológicos en los que los árboles forman parte, explícita o implícitamente, del objeto de estudio, presentaré diversos aspectos significativos de la etnoarqueología (algunos de la literatura, otros de mi propio trabajo de campo) para la construcción de nuevas preguntas dirigidas al registro arqueológico y la configuración de nuevos marcos de referencia para el ejercicio de la disciplina. Así, el principal objetivo es alimentar y enriquecer reflexiones críticas que hagan converger mediante la etnoarqueología objetivos, intereses y prácticas de la comunidad arqueológica interesada en las interacciones entre personas y árboles, entre sociedad y entorno, entre naturaleza y cultura.

\section{Prácticas invisibilizadas: aprovisiona- miento y consumo de combustible}

Si algo puede aportar la antracología al debate sobre la invisibilidad de los árboles en la literatura de las ciencias sociales es una ya madu- ra reflexión en torno a la relevancia material y social de diversas prácticas cotidianas en que la madera, presencia material del árbol, constituye un elemento central. Existen numerosas razones de carácter teorético para poner de relieve la escasa visibilidad de los árboles en la literatura de las ciencias sociales (Rival 1998; Jones y Cloke 2002; Watkins 2014), pero una de las más relevantes desde un punto de vista arqueológico, debido a su relevancia material, es la falta de atención sobre las prácticas de aprovisionamiento y consumo de combustible.

Desde la domesticación del fuego hasta la reciente y parcial generalización de los combustibles fósiles, la madera, biomasa vegetal de las plantas leñosas, constituye la principal fuente energética de la humanidad. Asimismo, los fragmentos de carbón, residuos materiales de este consumo energético, constituyen debido a su naturaleza favorable a la preservación en contextos arqueológicos, los más recurrentes restos de plantas en yacimientos de cualquier cronología en todo el planeta. Sin embargo, la atención a las actividades relacionadas con el aprovisionamiento y consumo de combustible es insignificante en la literatura arqueológica más allá de los propios trabajos antracológicos, a menudo presentados como anexos; es decir, trabajos realizados por especialistas y dirigidos a un público especializado dentro de la comunidad arqueológica, pero ajenos a las narrativas relacionadas con los aspectos sociales y culturales (Picornell-Gelabert y Servera-Vives 2017; Picornell-Gelabert et al. 2017).

El consumo de combustible vegetal leñoso - leña y/o carbón -, como toda práctica energética, se estructura en relación a modos de producción y consumo energético (Fernández y González 2014), prácticas que son a su vez estructuradas en relación a las interacciones que toda sociedad establece entre humanos y no-humanos (Horta et al. 2014: 115). Así pues, una aproximación arqueológica al consumo energético debe partir del reconocimiento de esta práctica socio-ambiental como superficie de interacción entre naturaleza y cultura. La percepción del entorno y las relaciones materiales con éste son diversas, variables y creativas en las culturas humanas (Descola 2012: 468). De esta forma, las complejas redes de interacción social que implica el consumo energético en la sociedad moderna capitalista no pueden ser extrapoladas a sociedades nooccidentales actuales o del pasado. Diferentes formas de percibir el entorno son indisolubles 
de diferentes maneras de relacionarse materialmente con éste, de estar en el mundo (Descola 2005).

Esto da lugar a diferentes maneras de estructurar las prácticas de aprovisionamiento y consumo de combustible a partir de diversas maneras de entender los árboles. El consumo de combustible se desarrolla de forma cotidiana en los hogares y contextos domésticos. En este sentido, pues, se trata también de una práctica coherente con la estructuración del resto de actividades de mantenimiento de los grupos humanos (Montón-Subías 2010; Hernando 2005). En este sentido, la recolección y consumo de leña constituye una arena social en la que estos modos relacionales (sensu Descola 2005), las formas diversas de entender y relacionarse materialmente con el entorno, son negociadas por los individuos de cada grupo social, socializadas, transmitidas a los hijos y perpetuadas como actividades domésticas recurrentes que identifican al conjunto social (Austin 2000; Picornell-Gelabert y Servera-Vives 2017).

Estas prácticas sociales relacionadas con el consumo energético están basadas en un conocimiento práctico transmitido generacionalmente mediante estas mismas acciones diarias que implican relación entre personas y plantas durante la recolección de la leña. Este proceso de aprendizaje, mediante el que se materializan los modos relacionales propios de cada sociedad, está integrado en rutinas y hábitos que son igualmente relevantes para el análisis del consumo energético (Horta et al. 2014: 117). Este proceso de aprendizaje se realiza en los ámbitos domésticos y en relación a las prácticas que el grupo desarrolla cotidianamente en el paisaje, permitiendo la transmisión generacional de ideas específicas de espacio, paisaje y naturaleza ligadas a la identidad del grupo social (Austin 2000; Picornell-Gelabert y Servera-Vives 2017).

La literatura arqueológica ha relegado los agentes que desarrollan estas prácticas cotidianas de aprovisionamiento y consumo de leña a una supuesta pasividad, disciplina y rutina específicamente orientada a la satisfacción funcional de una necesidad material. El consumo energético en ámbitos domésticos ha sido definido como una tarea tediosa, obscura, aborrecible y rutinaria (Heizer 1963: 189), practicada por agentes minorizados en los discursos históricos y arqueológicos (mujeres y niños, Montón-Subías 2010; Hernando 2005) y ajenos a la creatividad social. No obstante, es mediante estas prácticas que los agentes viven la experiencia de convenciones sociales relevantes para su identidad (De Certeau 1980) e intrínsecamente relacionadas con una determinada manera de entender y estar en el mundo (Descola 2005).

En este sentido, una de las aportaciones más significativas de la etnoarqueología es ofrecer un escenario de visibilización de estas complejidades y su relevancia social. El estudio etnoarqueológico de las relaciones entre personas y árboles/madera se convierte en un ejercicio mediante el cual estas cuestiones pueden salir de la sombra en que aproximaciones funcionalistas (en referencia a la acción) y androcéntricas (en referencia a los agentes) las han condenado al considerarlas prácticas rutinarias ajenas a la creatividad social. Asimismo, la etnoarqueología ofrece también la posibilidad de conjugar las prácticas de la comunidad antracológica con discursos y narrativas arqueológicas más amplios, más allá de especializaciones disciplinarias.

Los métodos y técnicas usados por los antracólogos han experimentado un notable desarrollo desde el inicio del siglo XXI, permitiendo ir más allá de la mera identificación taxonómica en el estudio de los restos arqueológicos de madera y carbón. Diversas actividades humanas en relación con los árboles quedan reflejadas en la anatomía de la madera, permitiendo analizar diámetros explotados (ramas, troncos), tipo de aprovechamiento de la biomasa vegetal (tala, recolección de leña muerta) y gestión del estrato arbóreo de los bosques (podas sistemáticas, irrigación) (Dufraisse 2008; Euba et al. 2015; Wrigth e. p.). Estos éxitos de laboratorio de la comunidad antracológica permiten avanzar en el estudio de la relaciones entre personas y árboles mediante el registro arqueológico. Es precisamente en este sentido que una aproximación etnoarqueológica, desde el presente, se convierte en especialmente oportuna, al ofrecer una posibilidad de diálogo entre las posibilidades técnicas de una disciplina concreta, la antracología, con aspectos y debates teóricos más amplios compartidos por la comunidad arqueológica en general.

\section{Etnoarqueología de los árboles y el com- bustible}

Si la comunidad antracológica constituye el colectivo arqueológico más sensibilizado res- 
peto a la relevancia de las prácticas de aprovisionamiento y consumo energético (o prácticamente el único), no es de extrañar que sea de este mismo colectivo del que han surgido los estudios etnoarqueológicos sobre madera y combustible. En los últimos 20 años han ido apareciendo de forma intermitente trabajos etnoarqueológicos en la literatura antracológica. Aunque se trata de trabajos con objetivos y metodologías muy diversas, la mayoría de ellos exploran el consumo energético - de leña - en contextos domésticos, ya que este tipo de conjuntos son los que centran la mayor atención de los propios estudios antracológicos. Así, si la investigación experimental, desarrollada en contextos controlados de laboratorio, ha permitido a la disciplina desarrollar herramientas más diversas y efectivas para la interrogación del registro antracológico, la etnoarqueología se va perfilando como un ámbito de reflexión en el que, a partir de la experiencia etnográfica, reflexionar sobre aspectos teóricos y metodológicos (Dufraisse et al. 2007; Picornell-Gelabert 2009a, b; Picornell-Gelabert et al. 2011, Zapta et al. 2003). Son este tipo de cuestiones las que, partiendo de una reflexión etnoarqueológica, ayudan a enriquecer las aproximaciones al registro antracológico y, a su vez, las narrativas arqueológicas sobre las interacciones entre humanos y plantas.

\subsection{Paleoeconomía del combustible}

El estudio del consumo de combustible arbóreo en grupos de cazadores-recolectores, que desarrollan modos relacionales significativamente distintos al occidental (Descola 2012), permite ilustrar como las diferentes percepciones del entorno y maneras de relacionarse con él implican también una relación material distinta con los árboles y los bosques. La economía del combustible constituye un tema fundamental en el desarrollo de la antracología desde los inicios de su configuración como disciplina arqueológica, vinculada a la arqueología paleolítica europea (Théry-Parisot 1998; Uzquiano 1992). Así, el estudio de la gestión del combustible en estos grupos ha dado lugar a algunos de los trabajos más inspiradores en los que se contrastan las premisas teóricas de la paleoeconomía de grupos cazadores-recolectores desarrollados en arqueología. Estos ejercicios (Piqué 1999, 2002; Caruso et al. 2008; Caruso 2010, 2014, 2015; Henry et al. 2009; Henry y Théry-Parisot 2014a, b; Manzi y Spi- kins 2008) han alimentado debates teóricos de la disciplina antracológica, y de la propia arqueología paleolítica, sobre la organización y significación social de la captación de recursos en estos grupos, atenuando muchas asunciones de carácter reduccionista o funcionalista.

En este sentido, un aspecto interesante es la propia concepción de recurso energético. Mediante el estudio de casos etnográficos se ha cuestionado la consideración de toda biomasa vegetal - leña - de un paleopaisaje como recurso energético explotable por parte de grupos cazadores-recolectores. Estudios detallados de casos etnográficos han puesto de relieve las complejas relaciones que se establecen entre personas, las diversas actividades que estas desarrollan y el entorno, demostrando que para que la madera de un árbol o un arbusto sea recolectada y consumida como leña deben cumplirse determinadas condiciones sociales que van más allá de su mera presencia en el entorno de un campamento de cazadores-recolectores (Piqué 1999). Estos trabajos etnoarqueológicos han cuestionado el estudio de la recolección de leña como una práctica de explotación del entorno que pueda explicarse per $s e$, en base a la biodiversidad (especies leñosas presentes en el entorno) o a las propiedades físico-químicas de la madera de cada especie, sin tener en cuenta su contexto social. Desde la consideración de la madera de determinados árboles y/o de una masa forestal concreta como una fuente energética explotable, hasta las prácticas diversas que acompañan los desplazamientos para su captación y la diversidad de usos y contextos de consumo de esta leña (con gran diversidad de significados sociales), existen complejas redes de interacción social de las que no se puede abducir la captación y consumo de combustible (Henry et al. 2009; Henry y Théry-Parisot 2014a, b).

Estos casos de estudio etnográficos, pues, evidencian como las prácticas de consumo energético en estos grupos, lejos de ser tareas simples, monótonas, tediosas y desconectadas de toda complejidad social, constituyen un elemento importante a la hora de aproximarnos a las complejas redes de interacción entre agentes humanos y no-humanos (Caruso, Mansur y Piqué 2008). Visibilizan igualmente el rol de los agentes de estas prácticas y su relevancia social, frecuentemente invisibles en las narrativas arqueológicas sobre sociedades cazadoras-recolectoras. En el caso de la leña, esta ha sido frecuentemente considerada un 
recurso recolectado y, por ende, femenino en la literatura arqueológica. Las complejidades y significación social del aprovisionamiento y consumo de combustible que reflejan estos trabajos etnoarqueológicos, así como la significación social de las prácticas femeninas en relación al mundo vegetal en las comunidades cazadoras-recolectoras, constituye otro de los aspectos relevantes que se han podido visibilizar con una base empírica (Piqué et al. 2008).

En este mismo sentido, el estudio de escenarios etnográficos ha permitido también complejizar y enriquecer la aproximación arqueológica al consumo energético en sociedades cazadoras-recolectoras. Si la recolección de leña está lejos de ser una tarea mecánica llevada a cabo por agentes pasivos y socialmente poco relevantes (mujeres y niños), el consumo de ésta en los hogares y estructuras de combustión tampoco puede considerarse desde una perspectiva simplista y mecanicista. Como la práctica totalidad de trabajos etnoarqueológicos sobre el combustible evidencian, el consumo de leña constituye una práctica relevante en las actividades cotidianas de mantenimiento de los grupos cazadores-recolectores. De esta misma forma, el rol social del fuego en la comunidad, es decir, el consumo de este combustible, tampoco puede ser reducido al desarrollo de actividades simplemente mecánicas.

\section{2. Árboles, combustible y construcción del espacio social}

\subsubsection{Entornos de abundancia}

Más allá de lo estrictamente referido a la economía del combustible, los estudios etnoarqueológicos permiten analizar en profundidad las relaciones entre las concepciones del paisaje y el significado de las prácticas sociales desarrolladas en relación a la vegetación. Si en el caso de las sociedades cazadoras-recolectoras la explotación del combustible no puede ser explicada como un comportamiento simplista y mecánico, en otro tipo de contextos sociales con una ocupación más o menos sedentaria del territorio y el desarrollo de prácticas productivas diversas, el consumo de combustible tampoco responde a estrategias simples de maximización y rendimiento en la explotación de recursos naturales para la producción energética (con toda la complejidad que este término entraña, Descola 2012: 457-459). Durante años, la propia literatura antracológica había intentado analizar (y hasta modelizar, p. ej. Shackleton y Prins 1992) la gestión de los recursos leñosos en sociedades no-occidentales en función de la cantidad de biomasa vegetal disponible, su biodiversidad y las características físico-químicas de la madera de cada especie, pero el propio desarrollo teórico-metodológico de la disciplina (Asouti y Austin 2005; Dufraisse 2012, 2014; Austin 2000; Johannessen y Hastorf 1990; PicornellGelabert y Servera-Vives 2017; Scheel-Ybert et al. 2016; Marconetto y Mafferra 2016) y la contribución a ello de la literatura etnoarqueológica (Dufraisse et al. 2007; Zapata et al. 2003; Picornell-Gelabert 2009a, b; PicornellGelabert et al. 2011), han puesto de relieve lo simplista e inoportuno de este tipo de aproximaciones.

Diversos estudios etnoarqueológicos han analizado la complejidad de la recolección y consumo de leña más allá de medir en el entorno de cada comunidad la abundancia o escasez de biomasa leñosa. Algunos de estos trabajos se han llevado a cabo en grupos que desarrollan una agricultura de roza y quema en ambientes tropicales y subtropicales, en los que la disponibilidad de recursos leñosos es especialmente elevada. En los pueblos yelemen de Papúa, por ejemplo, la obtención de leña depende de varios procesos productivos, como la apertura de nuevas rozas (vinculado a los ciclos agrícolas), la amortización de antiguas casas construidas con madera (en relación al entramado de toma de decisiones que implica el abandono y nueva fundación de hogares y la selección de maderas para la construcción) o la disponibilidad estacional de madera de deriva en ríos cercanos. Las zonas boscosas en las que se recoge la madera, los procesos de obtención y selección de los diámetros de la misma (desde ramas de varios tamaños hasta troncos) y los agentes de esta práctica (mujeres y niños $\mathrm{u}$ hombres adultos) dependen en gran medida del contexto de consumo. Así, las mujeres obtienen de los bosques considerados propios de los habitantes del pueblo la leña para uso cotidiano en los hogares, seleccionando a su vez los diámetros en función de la utilidad del combustible en cada caso. En cambio, son los hombres los encargados de recolectar la madera que va a utilizarse como combustible para calentar las piedras mediante las cuales se cocina en los hornos de cubeta. Este cambio de zonas de aprovisionamiento, de selección de diámetros y de los/las recolectores se deben, 
en este caso, al uso social de estos hornos, en los que se cocina la comida consumida en actos de comensalidad en los que se dirimen diversos aspectos sociales relevantes para la comunidad (Dufraisse et al. 2007).

De forma similar, el aprovisionamiento de combustible vegetal leñoso entre los fang de Guinea Ecuatorial se desarrolla en un ambiente de gran abundancia de biomasa vegetal, el bosque tropical centroafricano (Fig. 1). Lejos de aprovisionarse de combustible de forma azarosa, mecánica y despreocupada en un entorno con gran disponibilidad de recursos leñosos, como cabría esperar en función de modelos basados en relaciones de esfuerzo-rendimiento, las mujeres fang obtienen el combustible de forma diaria en función de una gran diversidad de variables sociales (detallado en PicornellGelabert 2009a, b; Picornell-Gelabert et al. 2011).

A pesar de estar rodeados de una impresionante masa boscosa, los fang obtienen la leña de lugares muy determinados. Su esquema topocéntrico de organización del espacio social, cuyo eje lo constituyen las casas de las familias que habitan en cada pueblo ("dzaá"), distingue entre zonas en que se cultivan vegetales y se obtiene leña para consumo doméstico, y zonas en las que estas actividades no se desarrollan. El principal aporte energético fang es un subproducto agrícola. Al abrir nuevas rozas para los huertos domésticos ("tsií) en función de los ciclos estacionales, los hombres de cada familia talan determinados árboles y arbustos para liberar espacio de cultivo. Esta actividad, una vez secada la madera y quemada la hojarasca, genera una reserva de combustible que es cuidadosamente seleccionada y organizada por las mujeres de la familia, también responsables de la plantación (Fig. 1a y b). Las nuevas rozas se efectúan en zonas de bosque maduro ("afán") o de bosque secundario en las que se han desarrollado periodos de barbecho más ("mbut") o menos ("ekot) dilatados que han permitido una regeneración de la vegetación.

Este stock de combustible es usado cotidianamente por las mujeres, que transportan la leña a diario del huerto a la casa en los mismos trayectos en los que trabajan los huertos y recogen sus productos (Fig. 1d). No obstante, existen momentos en que la demanda de combustible no puede ser satisfecha por estas reservas, al final de las estaciones del ciclo agrícola o debido a momentos de alta deman- da puntual, vinculados a celebraciones en las que se prepara una gran cantidad de yuca fermentada (proceso que requiere gran cantidad de combustible) y platos diversos que van a ser servidos durante las celebraciones. En estos casos, las mujeres pueden talar pequeños árboles de los huertos en barbecho ("ekot" o "mbut") que pertenecen a su familia, es decir, que han sido cultivados por ellas mismas o sus familiares (Fig. 1c y e). En ningún caso las mujeres recogen leña del bosque primario ("afán") que, aunque su familia ostente derecho de usufructo de una parte del mismo, nunca ha sido anteriormente modificado mediante trabajos agrícolas que parten de la tala de rozas, tarea realizada por los hombres de la familia. Asimismo, tampoco pueden acudir a zonas de barbecho ajenas o, si se ven obligadas a ello por necesidad, deben pedir permiso y negociar con la familia que trabajó esos huertos.

En cambio, existen otros momentos de consumo de combustible en que los agentes son distintos $\mathrm{y}$, consecuentemente, también lo son las zonas de aprovisionamiento. Es el caso del combustible consumido en los "abá", conocidos como casa de la palabra, espacios masculinos de los pueblos (generalmente porches con bancos de madera) en que se reúnen los hombres para dirimir cuestiones sociales o, simplemente, para pasar los ratos desocupados (Fig. 1f). En estos espacios se prenden hogares para calentarse de madrugada, iluminar de noche y preparar brebajes y comidas relacionados con actos ritualizados o de comensalidad estrictamente masculinos. En estos casos, en que el uso del fuego es puntual y no diario (como en las cocinas de las mujeres), el combustible es recolectado por los hombres del grupo también en espacios en que el bosque ha sido transformado mediante la actividad agrícola de la familia a la que pertenecen ("ekot" y "embut").

En cambio, cuando los hombres se encuentran en campamentos de caza en pleno bosque ("afán"), en los que pueden hacer estancias de varios días o incluso semanas, el combustible también es usado a diario para funciones diversas. A parte de iluminar las noches y ser usado para cocinar la comida de los cazadores, el fuego también es usado para ahumar la carne cazada y poderla mantener así varios días, hasta regresar al pueblo. En estos casos sí que se recolecta combustible del bosque primario en que se desarrollan las actividades cinegéticas. 


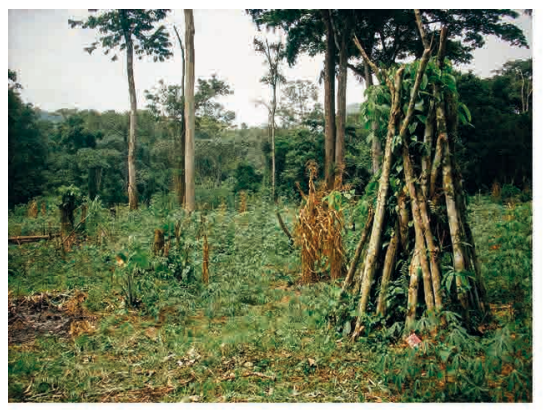

A

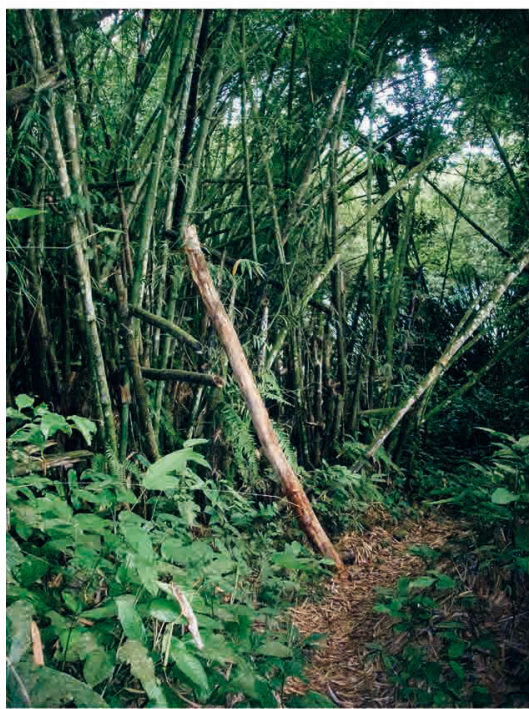

$\mathrm{C}$

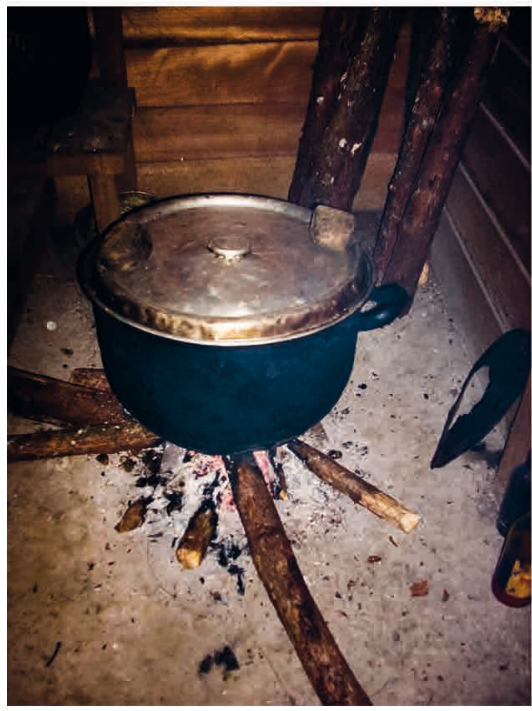

$\mathrm{E}$

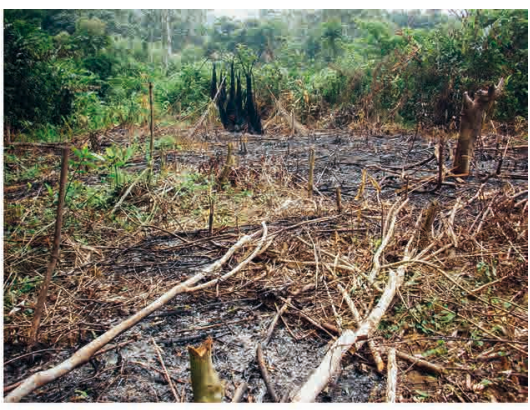

$\mathrm{B}$

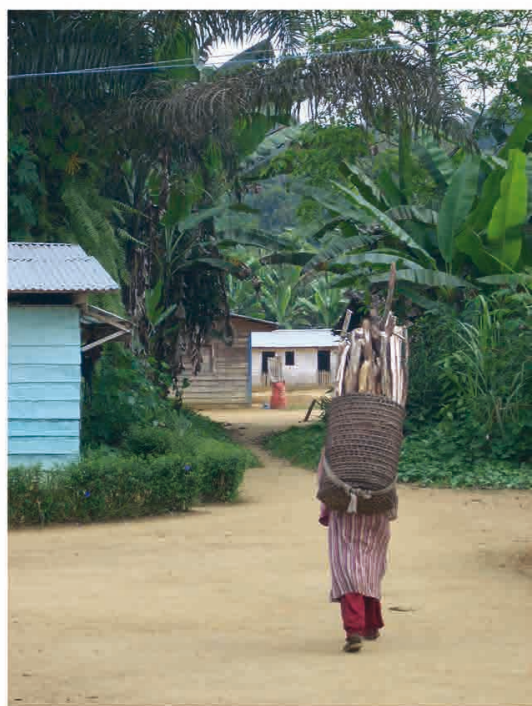

$\mathrm{D}$

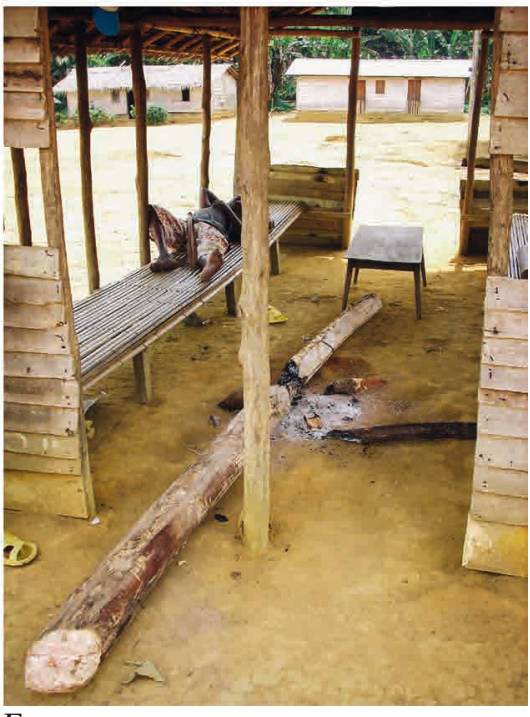

$\mathrm{F}$

Figura 1. Aprovisionamiento y consumo de leña entre los fang de Guinea Ecuatorial: a) Leña agrupada en un huerto ("tsií") después de la tala y quema, que es recogida a diario por las mujeres que trabajan y se aprovisionan del huerto; b) huerto talado y quemado, listo para empezar a cultivar; c) Tronco de un árbol ("asás", Macaranga barteri) talado en un barbecho ("ekot") y secándose para ser posteriormente usado como leña; d) Mujer fang transportando leña del huerto a al pueblo (“dzaá") para consumo doméstico; e) Hogar de una cocina con una olla de envueltos de yuca fermentada ("mbom mbog"); f) Hogar apagado en un "abá". Fotografías realizadas por el autor en Misergue en febrero de 2008. 
Así, la leña del "afán" es recolectada y consumida en el propio bosque, sin entrar en los espacios socializados (a partir de la transformación material de la vegetación mediante rozas) del esquema topocéntrico anteriormente mencionado; es decir, el pueblo ("dzaá"), los huertos ("tsié") o los barbechos ("ekot" o "mbut"). Asimismo, esta actividad es exclusivamente realizada por los hombres adultos, que son igualmente los únicos que se adentran en el "afán" a cazar y pescar. La construcción del espacio social fang, que se manifiesta a través de un paisaje abierto en que no existen más delimitaciones físicas que las propias derivadas de las actividades productivas (modificación de la vegetación para producción agrícola y construcción de casas), considera el bosque primario como un espacio habitado por una gran diversidad de agentes no-humanos: difuntos, árboles, animales y plantas con los que conviven los fang.

En cambio, cuando la leña es recolectada por las mujeres ésta procede de espacios considerados socializados mediante trabajos agrícolas ("ekot", "mbut" o "tsí1"), propios de la comunidad y en los que no pueden operar estos agentes. Igualmente, es consumida en este espacio social (en las cocinas del pueblo), en el núcleo topocéntrico ("dzaá”), en espacios propiamente humanos, en los que las personas vivas operan con cierta seguridad respeto a estos otros agentes que pueden entrañar riesgos sociales. En estos espacios seguros la comida puede ser producida, cocinada y consumida sin que existan interferencias de los agentes no-humanos con los que conviven los fang en un continuum social en que se integran personas vivas, difuntos y una diversidad de otros agentes que interfieren en la vida social y que, de no poder controlarlos, pueden ser problemáticos.

Esta concepción del paisaje y organización del espacio social opera en relación a los espacios en que se obtiene combustible en función de quien lo recolecta y en qué lugar va a ser consumido. Igualmente, esta manera de entender y socializar el paisaje también opera en relación a la selección de los combustibles consumidos. El bosque tropical alberga una gran biodiversidad y, en el caso de la vegetación leñosa, ofrece una gran diversidad de árboles y arbustos potencialmente explotables como combustible. Existen entre las mujeres fang opiniones comunes sobre las cualidades materiales como combustible de la madera de los diferentes árboles, unos considerados mejores que otros. Pero, en cualquier caso, esta consideración de buen combustible no opera como elemento selectivo en la recolección de leña. Es decir, cualquier madera que una mujer encuentre en el huerto después de la roza y quema del mismo estará seca $\mathrm{y}$, por tanto, constituye un combustible apto para ser consumido en la cocina. En ningún caso, una mujer fang irá a recolectar leña porque en el huerto solo queda madera de una especie que no considere el mejor combustible; es leña y puede ser usada como tal sin necesidad de más trabajos.

No obstante, sí que existen determinadas especies que nunca son usadas como combustible. Son el caso del "ebaiñ" (Pentaclethra macrophylla) y el "ñuará" (Tetrorchidium didymostemom), árboles que ostentan un carácter y una potencia de acción social que es vista como problemática por los fang. El "ebeiñ" es considerado un árbol violento que si es recolectado como leña, incorporado en el espacio social (la cocina en el pueblo) y consumido por los miembros de la familia a través de su uso como combustible para preparar la comida, va a causar problemas y estallidos violentos en el grupo. Igualmente, el "ñuará" es considerado un árbol con la capacidad de consumir, de pudrir la riqueza material de los humanos si, por el mismo procedimiento, es introducido en el espacio social y consumido. Si esto sucede, las riquezas materiales de la familia se verán gravemente mermadas (infertilidad de huertos, imposibilidad de tener descendencia, falta de éxito en la caza).

La selección de determinadas especies como combustible en función de las propiedades físico-químicas de su madera y/o su recurrencia en el entorno constituye un gran tema de debate en la literatura antracológica. No obstante, diversos estudios etnoarqueológicos, como el caso fang y otros (p. ej. Zapata et al. 2003; Dufraisse et al. 2007; Herny y Théry-Parisot 2014a, b; Henry et al. 2009), evidencian que se trata de una aproximación simplista al aprovisionamiento de combustible y consumo energético y que existen muchos otros aspectos, como los diámetros, el estado de la madera, la socialización de los espacios de captación o la percepción del paisaje y los árboles, que operan en este sentido. Es decir, el consumo de leña no responde a acciones mecánicas de optimización del esfuerzo-rendimiento en función de la facilidad de captación 
o las características físico-químicas de la madera de una u otra especie.

Diversos casos etnoarqueológicos, como el anteriormente reseñado, ilustran como las prácticas de aprovisionamiento y consumo energético se desarrollan en relación al complejo entramado de relaciones socio-ambientales, estructuradas a partir de concepciones diversas del entorno, de las plantas y del paisaje; y estructurantes a su vez de éstas mediante su práctica cotidiana, en la que se negocian y perpetúan estas categorías sociales. De la misma forma, el desarrollo de aproximaciones etnoarqueológicas pone también de manifiesto como el consumo energético constituye una arena social de interacción socio-ambiental cuyas prácticas son variadas en función de las diversas maneras de entender y estar en el entorno y de la historicidad y particularidad de cada cultura.

\subsubsection{Entornos de escasez}

Otra de las cuestiones que se ha trabajado desde la etnoarqueología en relación a las herramientas teórico-metodológicas de la antracología es la gestión de los recursos forestales en contextos de escasez de biomasa leñosa (Caruso 2010, 2014, 2015; Piqué 1999, 2002; Herny y Théry-Parisot 2014a, b; Henry et al. 2009; Joly et al. 2009; Zapata et al. 2003; Manzi y Spikins 2008). Si en la literatura antracológica/arqueológica han existido consideraciones simplistas y funcionalistas en relación al consumo de combustible en grupos cazadoresrecolectores (consumen de manera aleatoria lo que hay en el entorno debido a su escasa complejidad social), en grupos sedentarios y/o productores se ha considerado que la selección o no del combustible recolectado depende de la escasez/abundancia de biomasa vegetal en el entorno. Se han propuesto modelos predictivos que infieren que los grupos que habitan en contextos de abundancia leñosa practican una selección del combustible en función de las propiedades físicas de la madera de cada especie; mientras que en zonas de poca disponibilidad los humanos se ven forzados a recolectar cualquier leña disponible sin seleccionar (Shackleton y Prins 1992). Si los casos de los agricultores de rozas en zonas tropicales evidencian las complejidades de la gestión del combustible en contextos de abundancia de leña, los estudios etnoarqueológicos en zonas de escasa vegetación leñosa indican también que en estos casos tampoco operan estas fórmulas simplificadas.

Un caso ilustrativo es el de los konkomba y los anufo del noreste de Ghana (toda la información presentada fue recogida mediante observaciones de campo y entrevistas realizadas en los distritos de Saboba, junio de 2015, y Chereponi, febrero de 2016). En esta zona de vegetación de sabana, aunque escasos, los recursos leñosos son la principal fuente energética de los hogares konkomba y anufo. La leña es el único combustible usado en los hogares domésticos de las mujeres (esposas y/o hijas) de los "landlords" de cada "compound" familiar (Fig. 2b). En estos espacios el fuego es principalmente utilizado para transformar alimentos, cocinar para la familia y calentar agua para usos diversos.

Otro uso importante de leña en estos pueblos es como combustible en las cocciones cerámicas (Fig. 2c-f). A diferencia del caso anterior, este consumo energético es más puntual, ya que está vinculado a los ritmos estacionales de la producción alfarera, delimitados por diversos aspectos como la sucesión de épocas de lluvia, que limitan el acceso tanto al combustible como a las fuentes de arcilla debido a la abundancia de zonas inundadas. Otros elementos que delimitan la producción cerámica a lo largo del año son los trabajos agrícolas en determinadas estaciones, que ocupan casi todo el tiempo de trabajo de las alfareras, o la celebración de mercados regionales en los que se vende la producción cerámica.

En cualquier caso, cuando la actividad alfarera es llevada a cabo, el último eslabón de la cadena operativa es la cocción de las piezas confeccionadas. En estas cocciones se combinan dos tipos de combustible: uno de alta inflamabilidad, para prender y aumentar rápidamente la temperatura, compuesto por gramíneas secas recolectadas en el entorno de los pueblos, antiguos techos de paja amortizados como combustible y/o paja resultante de las cosechas (Fig. 2f); y otro de más lenta combustión que mantiene la temperatura durante el tiempo que dura la cocción, compuesto por leña y/o bostas secas de vaca del ganado de la familia (Fig. 2d y e).

La leña es recolectada por las mujeres konkomba y anufo durante todo el año. Esta proviene de las zonas de sabana consideradas propias de las familias que habitan en cada uno de los pueblos, donde recogen ramas secas o cortan ramas de los árboles, sin abatirlos 

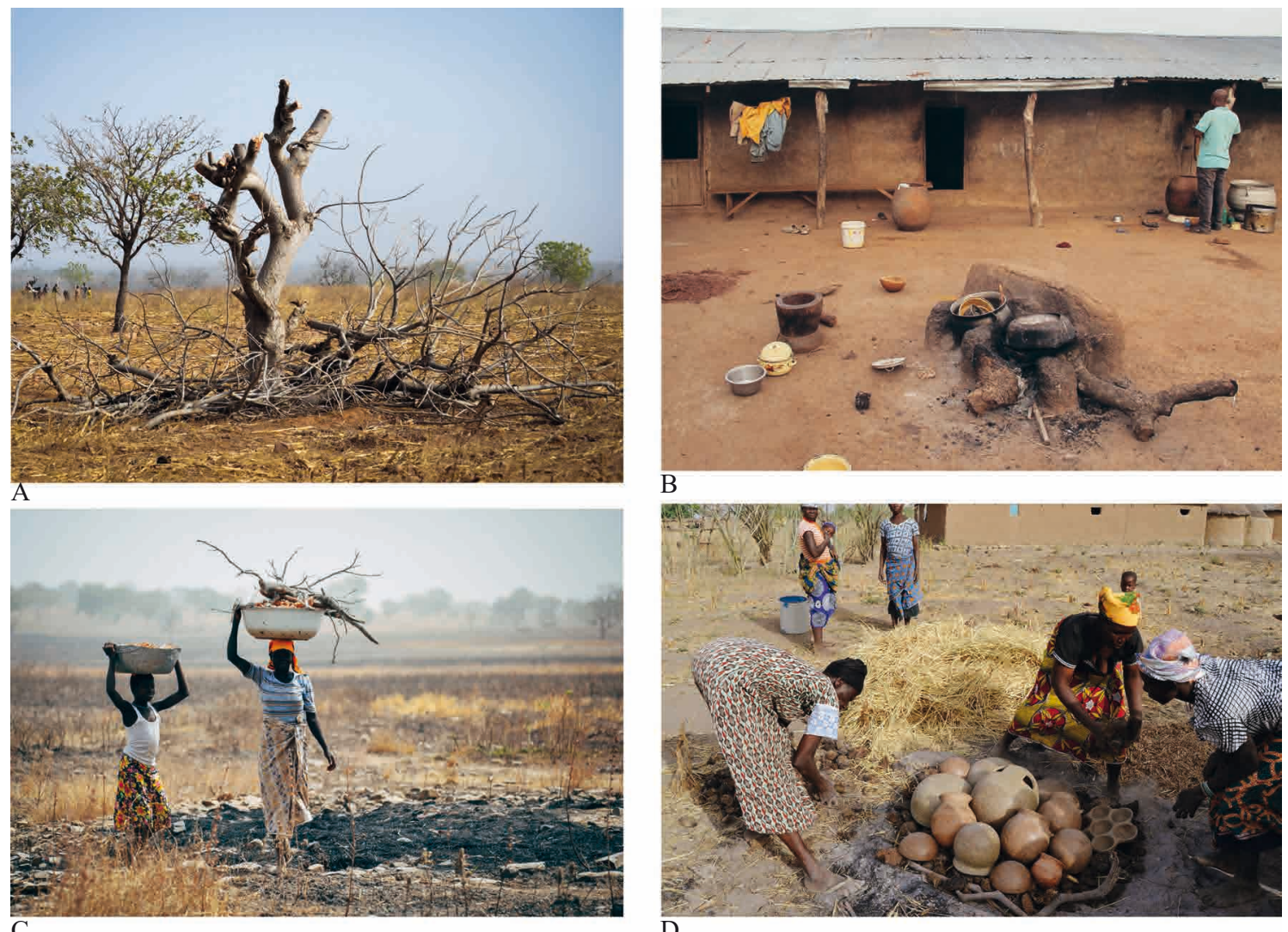

C

\section{D}

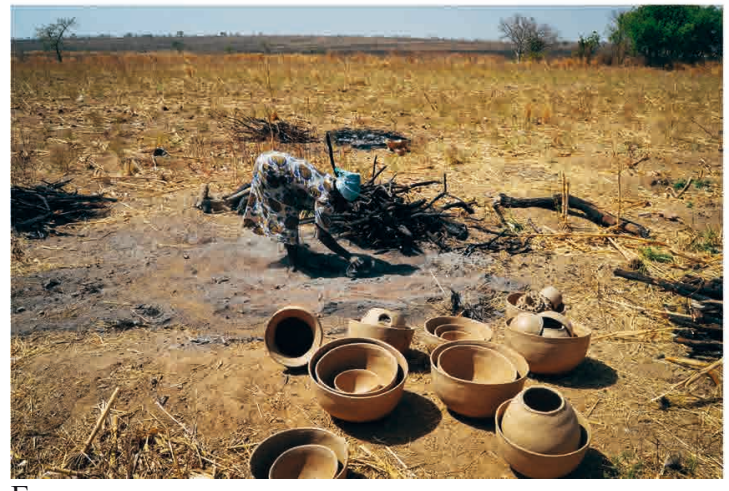

E

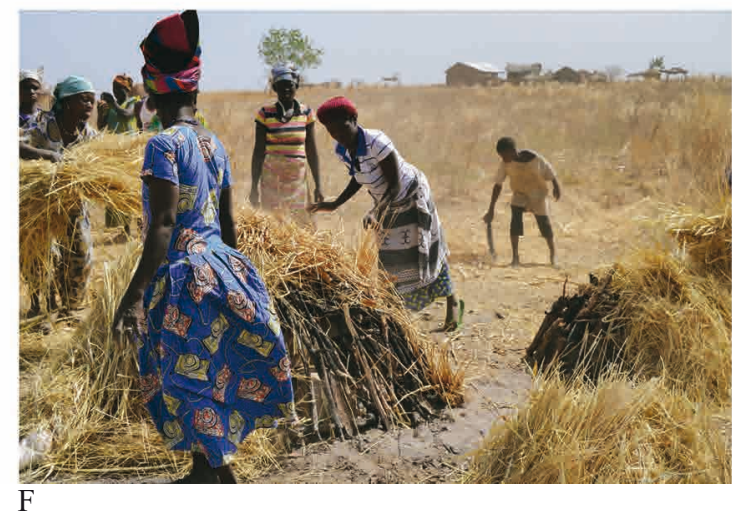

Figura 2. Aprovisionamiento y consumo de leña en los pueblos konkomba y anufo del noreste de Ghana: a) Árbol podado para la obtención de leña durante la época seca en los alrededores del pueblo de Nachen; b) Cocina exterior en uno de los "compounds" familiares del pueblo de Kuntuli; c) Transporte de arcilla y leña para una alfarera del pueblo de Nachen; d) Preparación de un horno para cocción cerámica en Nachen, en el que se usan como combustible bostas de vaca y leña; e) Preparación del combustible para una cocción cerámica en Nakaku, en que la leña es organizada por las alfareras en relación de su diámetro; f) Recubrimiento con paja de los cultivos del mismo horno cerámico de Nakaku. Fotografías del Grup de Recera ArqueoUIB tomadas en junio de 2015 y febero de 2016.

para que sigan produciendo leña en los años siguientes (Fig. 2a). La escasez de leña es percibida como uno de los principales problemas que condiciona la vida de estos pueblos. En muchos casos, las mujeres tienen que realizar grandes desplazamientos para conseguir leña (como pasa también con el agua) y, en época de lluvia, esta actividad está además condicionada por la inaccesibilidad de determinadas áreas inundadas y por la necesidad de reco- 
lectar con antelación para poder secar la leña antes de ser útil como combustible.

A pesar de estas limitaciones ambientales, la leña no es recolectada en cualquier lugar. Tanto los anufo como los konkomba sitúan en diversos puntos de su paisaje social lugares en los que habitan seres no-humanos con los que conviven y mantienen relaciones sociales. Estos lugares, que pueden ser zonas de ríos, pequeñas elevaciones, bosques o animales, constituyen emplazamientos en los que se llevan a cabo determinadas prácticas rituales ("shrines") que atañen a la familia, el clan o a todo el grupo étnico. En función de esta diversidad de prácticas ritualizadas operan normas sociales que delimitan el acceso a estos lugares y las actividades que en ellos se pueden desarrollar. Así, en estos bosques sagrados existen restricciones de acceso y desarrollo de determinadas actividades (Chouin 2002, 2008, 2009), como la caza de los hombres o la recolección de leña de las mujeres.

Así, este y otros estudios etnoarqueológicos (Caruso 2010, 2014, 2015; Piqué 1999, 2002; Herny y Théry-Parisot 2014a, b; Henry et al. 2009; Joly et al. 2009; Zapata et al. 2003) muestran como incluso en ambientes de escasez de biomasa leñosa, las prácticas sociales relacionadas con la leña se desarrollan en función de cómo el entorno es percibido, organizado socialmente y vivido, y no estrictamente en función de la escasez objetiva de leña en el entorno. Igualmente, estos factores conciernen a las relaciones materiales con los árboles y explican porque no cualquier árbol es apto para ser utilizado como combustible. Entre las mujeres anufo y konkomba hemos documentado la existencia de limitaciones de uso de determinadas especies como combustible. Como en el caso fang, las razones por las que un determinado árbol no es utilizado como combustible se relacionan con el carácter que se le reconoce. Muchos de estos árboles son invocados en diversas prácticas ritualizadas en las que se dirimen asuntos sociales relevantes. Así, estos no son utilizados en los hogares de las casas y cocinas, en las que se prepara el alimento que va a ser injerido por los miembros de la familia, para evitar agraviar a estos árboles a los que luego se les van a pedir mediaciones (para propiciar lluvias, por ejemplo) o para bloquear su capacidad de acción negativa en el seno del grupo.

No obstante, esta restricción de uso de determinados árboles como combustible opera en relación al contexto de consumo. Todas las especies de las que documentamos prohibiciones de uso en las cocinas dentro de las casas y "compounds" familiares pueden ser usadas, según las mujeres entrevistadas (alfareras o no), en las cocciones cerámicas. Esto se debe a la ubicación de los hornos alfareros y los hogares domésticos en relación a la construcción del espacio social konkomba y anufo. Si usar estas especies como leña en el interior de las casas y "compounds" entraña riesgos que afectan a todo el grupo familiar, su uso en los hornos cerámicos, ubicados fuera de estos espacios, no implica este peligro. Así, el escenario de consumo, el lugar en que se localiza el fuego que alimenta la leña, opera de manera directa en la gestión (rechazo de determinadas especies) del combustible leñoso. El elemento distintivo en este caso es la incorporación del árbol como agente en el espacio social (la casa) o su uso fuera de éste (en el horno cerámico).

\section{Visibilización de los árboles y de las prác- ticas cotidianas de consumo energético en las narrativas arqueológicas (reflexión final)}

Con este repaso a algunas de las cuestiones tratadas en diversos trabajos etnoarqueológicos sobre el aprovisionamiento y consumo de leña se visibilizan las diversas y complejas relaciones sociales que se establecen entre personas, paisajes y árboles. Con esta revisión he tratado solo algunos de los aspectos sociales que entraña el uso de biomasa vegetal leñosa para el consumo energético. Este conjunto de acciones constituyen arenas sociales de interacción entre lo humano y lo no-humano, un amplio campo de estudio en que la arqueología (y ya no solo la antracología) tiene la oportunidad de aportar reflexiones críticas partiendo de la materialidad a través del estudio de situaciones con el más amplio espectro espacio-temporal, desde la prehistoria al presente (Picornell-Gelabert et al. 2017).

El ejercicio etnoarqueológico en que consiste la aproximación a la materialidad de estas relaciones socio-ambientales y su significado social no puede resolver aspectos concretos de un caso arqueológico/antracológico determinado mediante una fotografia etnográfica del presente. No se puede resolver la interpretación de un diagrama o conjunto antracológico desde un caso etnográfico concreto (véase Picornell-Gelabert 2009a; Picornell-Gelabert et 
al. 2011). En cambio, sí que ofrece una doble oportunidad para la revisión crítica de los fundamentos heurísticos y herramientas teóricometodológicas con que la arqueología puede aproximarse al aprovisionamiento de leña y al consumo energético. Por una parte, estos estudios ofrecen la posibilidad de analizar en profundidad este tipo de interacciones materiales entre personas y vegetación/árboles/ paisaje, evitando definiciones simples y reduccionistas de las acciones y agentes involucrados basadas en apriorismos, contribuyendo así al reconocimiento, a partir de la base de la observación arqueológica del presente, de la relevancia social de los árboles y sus relaciones con los humanos. Por otra parte, permite a partir de esto cuestionar los útiles conceptuales con que la arqueología teoriza sobre las implicaciones materiales y sociales del uso y consumo de leña, con las que interpretamos estos aspectos en sociedades de un pasado más o menos remoto a partir del registro arqueológico.

Algunas de estas revisiones críticas de la aproximación arqueológica al consumo energético contribuyen al replanteamiento de una paleoeconomía del combustible que construya herramientas para ir más allá de una mera cuantificación del esfuerzo-rendimiento que su captación puede representar en un medio determinado. Este ejercicio permite redefinir el rol social de estas acciones, no analizándolas de forma aislada y partiendo de la base que sociedades simples desarrollan prácticas simples; es decir, sin ningún tipo de creatividad cultural y basadas exclusivamente en la resolución mecánica de necesidades materiales básicas. Así, los trabajos etnoarqueológicos orientados hacia estas problemáticas ayudan a repensar el análisis arqueológico de la captación de recursos en toda su complejidad, contemplando su interrelación con multitud de aspectos y procesos sociales.

Asimismo, diversos de los casos comentados muestran como las prácticas relacionadas con la captación de combustible participan activamente de la construcción y la experiencia de los paisajes sociales. La recolección de combustible no solo se ejerce en función a las delimitaciones que una sociedad hace de su entorno, sino que es (también) mediante estas prácticas diarias, cotidianas, que este espacio social es construido. Mediante el análisis de las complejas redes que operan entre las personas, los árboles y su entorno en la práctica diaria de recolección de leña, diversos casos de estudio etnoarqueológico evidencian como es también mediante estas prácticas cotidianas que las categorías a partir de las que un grupo construye su espacio social se materializan, son experimentadas y se perpetúan entre los miembros del grupo social. Es decir, que la práctica de aprovisionamiento y consumo energético y todos los agentes en ella implicados participan activamente en la construcción de un sentido de lugar, del paisaje social $\mathrm{y}$, en definitiva, de la construcción y experiencia de la identidad de los grupos humanos.

El estudio desde una perspectiva arqueológica de este tipo de interacciones socio-ambientales en el presente contribuye igualmente a la visibilización del rol social de los árboles y de las diversas y complejas relaciones que entre éstos y las personas se construyen a diario mediante el aprovisionamiento y consumo de leña. Así, estas aproximaciones etnoarqueológicas se convierten en una herramienta para la visibilización de la vida social de estos agentes no-humanos, un aspecto que va más allá de la práctica estrictamente antracológica o arqueológica, entroncando con aspectos teóricos y metodológicos más amplios de las ciencias sociales en relación a las complejidades de las interacciones entre naturaleza y cultura.

Con todo esto, este tipo de práctica etnoarqueológica se convierte igualmente en una herramienta para enriquecer, no solo las aproximaciones arqueológicas a estas cuestiones socio-ambientales, sino también la comunicación y discusiones conjuntas entre determinados especialistas de la arqueología ambiental $\mathrm{y}$ el resto de la comunidad arqueológica. $\mathrm{Si}$ la antracología dispone cada vez de un mayor número de técnicas y procesos de análisis de la anatomía de la madera para conocer los diámetros explotados, las diferentes prácticas de gestión de los árboles y los bosques (podas sistemáticas, talas, desmochado) o el estado de la madera en el momento de su consumo como combustible (madera verde o seca, almacenamiento de leña), estos casos etnoarqueológicos sirven de plataforma para evidenciar la relevancia social de estas cuestiones materiales y, así, hacer comprensibles complejos conjuntos de datos y sus tratamientos estadísticos a la comunidad de arqueólogos no familiarizados con ellos. Si la integración de perspectivas, prácticas y narrativas entre las conocidas como arqueología cientifica y arqueología teórica se presentaba como uno de los retos para la comunidad arqueológica ya a finales del si- 
glo XX (Albarella 2001; Pollard y Bray 2007; Jones 2001), puede que los avances técnicos que está experimentando la antracología en los últimos años junto con este tipo de trabajos etnoarqueológicos ofrezcan una buena oportunidad para un doble éxito en este sentido: (i) la visibilización de la relevancia y complejidad social de las prácticas de aprovisionamiento y consumo energético (ii) mediante la integración de aproximaciones y perspectivas entre la comunidad arqueológica más allá de especialidades concretas.

\section{Agradecimientos}

Quiero expresar mi gratitud a las personas entrevistadas en Ghana y Guinea Ecuatorial por ofrecerme la posibilidad de aprender de ellos, como antracólogo, arqueólogo y, también, como persona. Alexa Dufraisse y Ethel Allué Martí han compartido también sus conocimientos conmigo mediante discusiones que, de una u otra forma, están reflejadas en éste artículo. La primera de ellas también ha revisado el manuscrito. Agradezco también a los dos revisores anónimos sus comentarios para mejorar el texto. Cualquier error es responsabilidad únicamente mía.

\section{Bibliografía}

Albarella, U. (ed.) (2001): Environmental archaeology: Meaning and Purpose. Kluwe Academic Publishers, Dorddrecht.

Asouti, E.; Austin, P. (2005): Reconstructing woodland vegetation and its exploitation by past societies, based on the analysis and interpretation of archaeological wood charcoal macroremains. Environmental Archaeology, 10: 1-18.

Austin, P (2000): The emperor's new garden: woodland, trees and people in the Neolithic of southern Britain. Plants in Neolithic Britain and Beyond (Fairbairn, A.S., ed.), Oxbow, Oxford: 63-78.

Caruso, L. (2010): Ethnographie, archéobotanique et experimentation sur le site d'Ewan I (Tierra del Fuego, Argentine). Anthropobotanica, 1(5): 3-17.

Caruso, L. (2014): El uso de la Madera entre los cazadores-recolectores Selk'Nam. Cazadores de mar y tierra. Estudios recientes en arqueología fueguina (J. Oría, A.M. Tivoli, eds.), Editora Cultural Tierra del Fuego, Ushuaia: 335-359.

Caruso, L. (2015): Modalidades de adquisición y usos de la madera en sociedades cazadoras-recolectoras patagónicas. CSIC-IMF, Barcelona.

Caruso, L.; Mansur, M.E.; Piqué, R. (2008): Voces en el bosque: el uso de recursos vegetales entre cazadoresrecolectores de la zona central de Tierra del Fuego. Darwiniana, 46(2): 202-212.

Chouin, G. (2002): Sacred groves in history: Pathways to the social shaping of forest landscapes in Coastal Ghana. IDS Bulletin, 33(1): 39-46.

Chouin, G. (2008): Archaeological perspectives on sacred groves in Ghana. African sacred groves. Ecological dynamics and social change (M. Sheridan, C. Nyamweru, eds.), James Currey Ltd., Oxford: 178-194.

Chouin, G. (2009): Forests of power and memory: An archaeology of sacred groves in the Eguafo polity, Southern Ghana (c. 500-1900 A.D.). Anthropology - Dissertations. Paper 9.

Bradley, R. (2005): Ritual and domestic life in Prehistoric Europe. Routledge, London.

De Certeau, M. (1980): L'Invention du Quotidien. Vol. 1, Arts de Faire. Union générale d'éditions, París.

Descola, Ph. (2005): Par-delà nature et culture. Gallimard, París.

Descola, Ph. (2012): Beyond nature and culture. Forms of attachment. Journal of the Ethnographic Theory, 2(1): 447-471.

Descola, Ph.; Pálsson, G. (eds.) (2001): Naturaleza y sociedad. Perspectivas antropológicas. Siglo XXI, México.

Dufraisse, A. (2008): Firewood management and woodland exploitation during the Late Neolithic at Lake de Chalain (Jura, France). Vegetation History and Archaeobotany, 17: 199-210.

Dufraisse, A. (2012): Firewood and woodland management in their social, economic and ecological dimensions: new perspectives. Saguntum extra, 13: 65-74.

Dufraisse, A. (2014): Relation entre modes de collecte du bois de feu et état du milieu forestier : essai d'application du principe du moindre effort. Entre archéologie et écologie, une Préhistoire de tous les milieux. Mélanges offerts à Pierre Pétrequin (R.M. Arbogast, A. Richard, dir.), Presses univesitaires du Franche-Comté, Besançon: 493-504. 
Dufraisse A.; Pétrequin, A.M.; Pétrequin, P. (2007): La gestion du bois de feu: un indicateur des contextes socio-écologiques. Approche ethnoarcheologique dans les Hautes Terres de Papua (Nouvelle-Guinée indonésienne. Sociétés néolithiques. Des faits archéologiques aux fonctionnements socio-économiques (M. Besse, ed.), Actes du 27 e colloque interrégional sur le Néolithique (Neuchâtel, octobre 2005), Cahiers d'archéologie romande, vol. 108, Lausane: 115-126.

Elden, S. (2006): Heidegger's animals. Continental Philosophy Review, 39: 273291.

Euba, I.; Allué, E.; Burjachs, F. (2015): Wood uses at El Mirador Cave (Atapuerca, Burgos) based on anthracology and dendrology. Quaternary International Online first.

Fernández, R.; González, L. (2014): En la espiral de la energía Volumen I: Historia de la humanidad desde el papel de la energía (pero no solo). Libros en Acción/Baladre, Madrid.

Hastorf, C. (2009), Agriculture as Metaphor of the Andean State. Polities and Power: Archaeological Perspectives on the Landscapes of Early States (S.E. Falconer, C.L. Redman, eds.), University of Arizona Press, Tucson: 52-72.

Heizer, R.F. (1963): Domestic fuel in primitive society. Journal of the Royal Anthropological Institute of Great Britain and Ireland, 93: 186-194.

Henry, A.; Théry-Parisot, I.; Voronkova, E. (2009): La gestion du bois de feu en foret boreale: archéoanthracologie et ethnographie (Région de l'Amour, Sibérie). Gestion des combustibles au paléolithique et au mésolithique. Nouveaux outils, nouvelles interpretations (I. Théry-Parisot, S. Costamagno, A. Henry, ed.), BAR International Series 1914, Archaeopress, Oxford: 17-37.

Henry, A.; Théry-Parisot, Y. (2014a): From Evenk campfires to prehistoric hearths: charcoal analysis as a tool for identifying the use of rotten wood as fuel. Journal of Archaeological Science, 52: 321-336.

Henry, A.; Théry-Parisot, Y. (2014b): Fuel use management during the Mesolithic: recent approaches in archaeobotany. Tehcniques and terrotioires. New insights into Mesolothic Cultures (A. Henry, B. Marquebielle, L. Chesnaux, S. Michel, dir.), Paleoethnology, Toulousse: 65-83.

Hernando, A. (2005): ¿Por qué la Historia no ha valorado las actividades de mantenimiento? Dones $i$ activitats de manteniment en temps de canvi (P. González, S. Montón, M. Picazo, eds.), Treballs d'Arqueologia 11, Universitat Autònoma de Barcelona, Bellaterra: 115-133.

Hicks, D. (2016): The temporality of landscape revisited. Norwegian Archaeological Review, 49(1): 5-22.

Horta, A.; Wilhite, H.; Schmidt, L.; Bartiaux, F. (2014): Socio-Technical and Cultural Approaches to Energy Consumption. An introduction. Nature and Culture, 9(2): 115-121.

Inglis, D.; Bone, J. (2006): Boundary Maintenance, Border Crossing and the Nature/Culture Divide. European Journal of Social Theory, 9: 272.

Ingold, T. (1988): What is an animal? One World Archaeological Series $\mathrm{n}^{\circ} 1$, Unwin Hyman, London.

Ingold, T. (2000): The perception of the environment: essays on livelihood, dwelling and skill. Routledge, London.

Ito, M. (2008): Seeing Animals, Speaking of Nature Visual Culture and the Question of the Animal. Theory, Culture and Society, 25(4): 119-137.

Johannessen, S.; Hastorf, C. (1990): A history of fuel management (A.D. 500 to the present) in the Mantaro Valey, Peru. Journal of Ethnobiology, 10(1): 61-90.

Joly, D.; March, R.; Marguerie, D.; Yacobaccio, H. (2009): Gestion des combustibles dans la province de Jujuy (Puna, Argentine) depuis l'Holocène Ancien: croisement des résultats ethnologiques et anthracologiques. Gestion des combustibles au paléolithique et au mésolithique. Nouveaux outils, nouvelles interpretations (I. Théry-Parisot, S. Costamagno, A. Henry, ed.), BAR International Series 1914, Archaeopress, Oxford: 39-52.

Jones, A. (2001): Archeological theory and scientific practice. Cambridge University Press, Cambridge.

Jones, O.; Cloke P. (2002): Tree Cultures: The Place of Trees, and Trees in their Place. Berg, Oxford.

Latour, B. (1991): Nous n'avons jamais été modernes. Essai d'anthropologie symétrique. La Découverte, Paris.

Luff, R.; Rowley-Conwy, P. (1994): The (Dis)integration of Environmental Archaeology. Whither Environmental Archaeology (R. Luff, P. Rowley-Conwy, eds.), Oxbow Monograph 38, Oxbow, Oxford: 1-4.

Marconetto, B.; Mafferra, L. (2016): Todos los fuegos, el fuego: discusión en torno a las categorías modernas en la interpretación de registros antracológicos en contextos prehispánicos y coloniales. Cadernos do Lepaarq, 13(25): 460-483.

Montón-Subías, S. (2010): Maintenance Activities and the Ethics of Care. Situating gender in European archaeologies (L.H. Dommasnes, T. Hjørungdal, S. Montón-Subías, M. Sánchez Romero, N.L. Wicker, eds.), Archaeolingua Fundation, Budapest: 23-33.

Palmer, C.; Van der Veer, M. (2002): Archaeobotany and the social context of food. Acta Palaeobotanica, 42 (2): 195-202.

Philo, C.; Wilbert, C. (2000): Animal spaces, beastly places. An introduction. Animal Spaces, Beastly Places: New Geographies of Human-Animal Relations (C. Philo, C.Wilbert, eds.), Routledge, Florence: 1-36. 
Picornell-Gelabert, Ll. (2009a): Antracología y Etnoarqueología. Perspectivas para el estudio de las relaciones entre las sociedades humanas y su entorno. Complutum, 20(1): 133-151.

Picornell-Gelabert, Ll. (2009b): Quemar la prosperidad. Leña y vida cotidiana en los pueblos Fang de Guinea Ecuatorial. Afro-Hispanc Review, 28(2): 43-56.

Picornell-Gelabert, Ll.; Asouti, E.; Allué, E. (2011): The ethnoarchaeology of firewood management in the Fang villages of Equatorial Guinea, central Africa: Implications for the interpretation of wood fuel remains from archaeological sites. Journal of Anthropological Archaeology, 30: 375-384.

Picornell-Gelabert, Ll.; Servera-Vives, G. (2017): Landscape practices and everyday life in domestic spaces in Bronze Age Mallorca (Balearic Islands): perspectives for an archaeology of fuels. Quaternary International, 431(A): 73-89.

Picornell-Gelabert, Ll.; Allué, E.; Courty, M.A. (2017): An archaeology of fuels: social and environmental factors in behavioural strategies of multi-resources management, Quaternary International, 431(A): $2-5$.

Piqué, R. (1999): Producción y uso de combustible vegetal: una evaluación arqueológica. Treballs d'etnoarqueologia 3, Bellaterra, Universitat Autònoma de Barcelona-CSIC.

Piqué, R. (2002), Paisaje y explotación de los recursos forestales entre los canoeros magallánico-fueguinos. Aportación de la etnoarqueología. América Latina. Historia y sociedad, una visión interdisciplinaria. Cuatro años de "Aula Oberta” en la UAB (R. Piqué, M. Ventura, eds.), UAB, Bellaterra: 77-94.

Piqué, R.; Vila, A.; Berihuete, M.; Mameli, L.; Mensua, C.; Moreno, F.; Toselli, A.; Verdún, E., D. Zurro (2008): El mito de la "Edad del Piedra": Los recursos olvidados. Mujeres y Arqueología. Nuevas aportaciones desde el materialismo histórico. Homenaje al profesor Manuel Carrilero Millán (T. Escoriza, M ${ }^{a}$ López, A. Navarro, coord.), Junta de Andalucía, Granada: 59-104.

Pollard, A.M.; Bray, P. (2007): A Bicycle Made for Two? The Integration of Scientific Techniques into Archaeological Interpretation. Annual Review of Anthropology, 36: 245-259.

Rival, L. (1998): The Social Life of Trees. Anthropological Perspectives on Tree Symbolism (Materializing Culture). Berg, London.

Scheel-Ybert, R.; Fernandes, C.; Waisman, L. (2016): Of forests and gardens: landscape, environment, and cultural choices in Amazonia, southeastern and southern Brazil from c. 3000 to $300 \mathrm{cal}$ yrs BP. Cadernos do Lepaarq, 13(25): 424-458.

Shackleton, C.M.; Prins, F. (1992): Charcoal analysis and the "Principle of least effort": a conceptual model. Journal of Archaeological Science, 19: 631-637.

Théry-Parisot, I. (1998): Économie des combustibles et paléoécologie en contexte glaciaire et périglaciaire, Paléolithique moyen et supérieur du sud de la France. Anthracologie, expérimentation, taphonomie. Tesis Doctoral Inédita, Université París 1.

Théry-Parisot, I.; Chabal, L.; Chrzavzez, J. (2010): Anthracology and taphonomy, from wood gathering to charcoal analysis. A review of the taphonomic processes modifying charcoal assemblages, in archaeological contexts. Palaeogeography, Palaeoclimatology, Palaeoecology, 291(1-2): 142-153.

Uzquiano, P. (1992): Recherches anthracologiques dans le secteur Pyréneon-Cantabrique (Pays Basque, Cantabria et Asturias): Environnements et rélations homme-milieu au Pléistocène supérieur et début de l'Holocène. Tesis Doctoral, Université de Montpellier II.

Watkins, C. (2014): Trees, woods and forests. A social and cultural history. The University of Chicago Press, Chicago.

Wright, N. (en prensa): Examining dendrological features of oak as possible signals of systematic woodland management in the central Anatolian Bronze and Iron Ages. Quaternary International.

$\mathrm{Wu}$, J. (2010): Landscape of culture and culture of landscape: does landscape ecology need culture? Landscape ecology, 25: 1147-1150.

Zapata, L.; Peña, L.; Ibañez, J.J.; González, J.E. (2003): Ethnoarchaeology in the Morocan Jebala (Western Riff): Word and duna as fuel. Food, Fuel and Fields. Process in African Archaeobotany (K. Neumann, A.I. Butler, S. Kahlheber, eds.), Barth-Institut-Hendrik, Köln: 163-175. 